\title{
Competencia y arbitraje fiscal en la Unión Europea
}

\author{
Ramón Bonell \\ Universidad Complutense
}

\begin{abstract}
Resumen
Es innegable la preponderancia que ejerce en la sociedad la Unión Europea, y las distintas nociones efectuadas respecto de esta unión que asocia a los Estados miembros, y a los que desean adherirse. Derecho y Estado de bienestar son las bases fundamentales sobre las que se asienta el edificio jurídico de la sociedad. En sus distintas manifestaciones los ciudadanos buscan y desean el progreso y el desarrollo económico. Los intereses en conflicto que está viviendo la sociedad europea determinan la aplicación de los principios constitucionales en interés de los ciudadanos europeos. Estas divergencias en los enfoques de cada uno de los países miembros de la UE revelan las dificultades que vive el continente europeo.
\end{abstract}

Palabras clave: UE, competencia, arbitraje fiscal, deberes, obligaciones.

Clasificación JEL: $K, K 2$.

\begin{abstract}
It is undeniable that the preponderance society holds the European Union, and the various notions made in respect of this union associating the Member States, and to those who wish to join. Law and the welfare state are the fundamental basis on which the legal edifice of society rests. In its various manifestations citizens seek and desire progress and economic development. Conflicting interests that European society is living determine the application of constitutional principles in the interests of European citizens. These differences in the approaches of each of the EU member countries reveal the difficulties experienced by the European continent.
\end{abstract}

Keywords: EU, competence, arbitrage, duties, obligations.

JEL classification: $K, K 2$.

\section{Fiscalidad y globalización}

En la revolución de la economía digital, prácticamente toda la economía en el siglo XXI, requiere del tratamiento de la información, de los datos, la transparencia de la información financiera, siendo una función esencial el asesoramiento jurídico y tributario. En un entorno económico en el que se llevan a cabo operaciones vinculadas, que se ven afectadas por los precios de transferencia, dentro de la planificación fiscal de las empresas ${ }^{1}$.

${ }^{1}$ Cfr. AHMADOV, J. (2011). «The "Most Appropriate Method" as the New OECD Transfer Pricing Standard: Has the Hierarchy or Methods Been Completely Eliminated?», International Transfer Pricing Journal, 18 (3), 184. ATO (2005). «Australian Taxation Office Applying the Arm's-length Principle», Guide NAT 2726-04.2005; disponible en: http://www.transferpricing.com/pdf/Australia_Applying_arms_length_ principle.pdf. 
La globalización de la economía supone una transformación profunda de la división internacional del trabajo en el comercio exterior, de la configuración de los flujos financieros internacionales y las inversiones directas extranjeras y de las corrientes migratorias del capital humano, auténtico capital intelectual, todo lo cual cuestiona las ventajas de localización que Europa había disfrutado en el pasado².

AVI-YONAH, R. (1995). «The Rise and Fall of Arm's Length: A Study in the Evolution of US International Taxation», Virginia Tax Review, vol. 15, pp. 89-159. AVI-YONAH, R. (2010). «Between Formulary. Apportionment and the OECD Guidelines: A Proposal for Reconciliation», World Tax Journal, vol. 2, núm. 1. COOPER, J. y AGARWAL, R. (2011). «The Transactional Profit Methods in Practice: A Survey of APA Reports», International Transfer Pricing Journal, vol. 18, núm. 1. CORONADO,L.; CHEUNG, P. y KYTE, J. (2010). «An Overview of Arm's Length Approaches to Thin Capitalization», International Transfer Pricing Journal, vol. 17, núm. 4. DOUVIER, P. (2003). «Transfer Pricing and Research and Development», International Transfer Pricing Journal, nov./dic., pp. 218-221. DRAKE, J.; RODE, A. y WRIGHT, D. R. (2005). «IRS APA Initiatives», International Transfer Pricing Journal, septiembre/octubr, pp. 210-216. DUJSIC, M. (2008). «Digesting the Glaxo Decision: A Difficult Pill to Swallow for Transfer Pricing Practitioners», International Transfer Pricing Journal, sep./oct. FRANCESCUCCI, D. L. P. (2004). «The Arm's Length Principle and Group Dynamics», Part 1: The Conceptual Shortcomings, International Transfer Pricing Journal, marzo/abril, pp. 55-75. FRANCESCUCCI, D. L. P. (2004). «The Arm's-Length Principle and Group Dynamics - Part 2: Solutions to Conceptual Shortcomings», International Transfer Pricing Journal, nov./dic., pp. 235-246. FRIS, P. (2003). «Dealing with Arm's Length and Comparability in the Years 2000», International Transfer Pricing Journal, nov./dic., pp. 194-201. GILL, S. (2011). «Intangibles and Transfer Pricing: The Perils Faced by Multinationals in India», International Transfer Pricing Journal, vol 18, núm. 1. GOUTHIERE, B. (2011). «Key Practical Issues in Eliminating the Double Taxation of Business Income», Bulletin for International Taxation, vol. 65, núm. 4-5. HAMAEKERS, H. M. A. (2005). «Income Allocation in the 21st Century: The End of Transfer Pricing? Introductory Speech», International Transfer Pricing Journal, may./jun., pp. 95-102. HAMMER, R. (1993). «Will the Arm's Length Standard Stand the Test of Time? The Spectre of Apportionment, en K. Alpert y K. VAN RAAD (eds.), Essays on International Taxation, Holanda, Kluwer Law International. HELLERSTEIN, W. (2005). «Income Allocation in the 21st Century: The End of Transfer Pricing? The Case for Formulary Apportionment», International Transfer Pricing Journal, may./ jun., pp. 103-111. JOSEPH, A. (2005). «International Financial Reporting Standards and Thin Capitalization», International Transfer Pricing Journal, sep./oct., pp. 217-223. JOSEPH, A. «Australia - Increasing Role of Advance Pricing Agreements», International Transfer Pricing Journal, vol. 18, núm. 4. LENZ, M. y VOGELE, A. (1999). «A Case Study: What's in the Black Box», International Tax Review, 1999. MEHAFDI, M. (2000). «The Ethics of international Transfer Pricing», The Journal of Business Ethics, diciembre 2000, vol. 28, parte 2, pp. 365-381. NIAS, P. y IVINSON, J. (2002). «Back to Basics - Transfer Pricing Part 1 - The International Context», Tax Journal, 4 nov. OECD (2005). Are the Current Treaty Rules for Taxing Business Profits Appropriate for E-Commerce? París, disponible en http://www.oecd.org/document/. OECD (2007). Improving the Resolution of Tax Treaty Disputes, Committee on Fiscal Affairs, París, disponible en http://www.oecd.org/dataoecd/17/59/38055311.pdf. OECD (2008). «Transactional Profit Methods», Discussion Draft for Public Consultation. OECD (2008). Centre for Tax Policy and Administration 25 January 2008; OECD (2010). Transfer Pricing Guidelines for Multinational Enterprises and Tax Administrations, París. OOSTERHOFF, D. y WINGERTER, B. (2011). «The New OECD Guidelines: The Good, the Bad and the Ugly», International Transfer Pricing Journal, vol. 18, núm. 2. PEREIRA, M. y ZAMBUJAL-OLIVEIRA, J. (2011). «Transfer Pricing Analysis: The Case of a Manufacturing Company», International Transfer Pricing Journal, vol. 18, núm. 1. PICCIOTTO, S. (1992). International Business Taxation, Londres, Butterworths. RECIHART, T. A. y WRIGHT, D. R. (2006). «Proposed Cost Sharing Regulations: A Departure from Arm's Length?», International Transfer Pricing Journal, ene./feb., pp. 2-7. STIRLING, M. (2002). «Global Attitudes Towards Transfer Pricing», Tax Journal, junio; United States Internal Revenue Service Development of IRC Section 482 cases Ch. 61 Internal Revenue Manual, disponible en http://www.irs.gov/ irm/part4/ch46s03.html. VANN, R. J. (2010). «Taxing International Business Income: Hard-Boiled Wonderland and the End of the World», World Tax Journal, vol. 2, núm. 3, pp. 291-346. WUNDISCH, K. (2003). «Pharmaceutical Industry and Transfer Pricing: Anything Special?», International Transfer Pricing Journal, nov./dic., pp. 204-210.

2 DONGES, J. B. (2007). Estudios de Política Económica (1998-2006), Madrid, Unión Editorial. 
Las nuevas tecnologías de la información y la comunicación han reducido dramáticamente los costes de transacción económica, dando lugar a nuevas formas de producir y de trabajar en casi todos los sectores, y muy especialmente en el de los servicios profesionales.

En las últimas décadas se ha producido una profunda aceleración en la internacionalización del comercio, particularmente desde la entrada de China en la OMC en 2001. Como señala Xavier Gimbert²: «la internacionalización no es más que otra de las opciones que puede tener una compañía para crecer».

España en un principio fue reacia a embarcarse en este proceso de expansión comercial, puesto que el proteccionismo de nuestra legislación le permitía disfrutar de un mercado interior sin competencia exterior. En cambio, el ingreso de España en la UE en 1986 y las dificultades económicas le han obligado a acudir a los mercados transnacionales y «mirar hacia el exterior» para salir adelante.

Siguiendo a Daniel Lacalle ${ }^{4}$, el mercado rentabiliza el patrimonio de los ciudadanos, generan valor los mercados, autonomía financiera, la riqueza en los mercados se crea para todos, de tal forma que hay que leer los números, eso es lo que hacemos con la documentación de las operaciones vinculadas, reflejando los precios de transferencia, precios que responden a la idea de creación de valor, a la realidad empresarial y no al traslado de bases imponibles por criterios de conveniencia.

\subsection{Beneficios empresariales}

La tributación del beneficio empresarial ${ }^{5}$ ha de seguir a la creación de valor, poniendo la sustancia de lo que crea valor allí donde desea que tribute el beneficio ${ }^{6}$.

${ }^{3}$ Vid. GIMBERT, X. (2006). El enfoque estratégico de la empresa, Barcelona, Deusto, p. 155.

${ }^{4}$ LACALLE, D. (2013). Nosotros, los mercados. Qué son, cómo funcionan y por qué resultan imprescindibles, Barcelona, Deusto.

${ }^{5}$ Cfr. BRAITHWAITE, J. (2005). Markets in Vice, Markets in Virtue, Reino Unido, Oxford University Press. BRAITHWAITE, V. (2003). Taxing Democracy: Understanding Tax Avoidance and Evasion, Reino Unido, Ashgate. CNOSSEN, S. (1989). «Value Added Tax: Key to a Better Tax Mix», Australian Tax Forum, vol. 6, núm. 3, pp. 265-281. FENNELL, C. C. y FENNELL, L. A. (2003). «Fear and Greed in Tax Policy: A Qualitative Research Agenda», Washington University Journal of Law and Policy, vol. 13, p. 75. GRANWELL, A. y MCGONIGLE, S. (2006). «US Tax Shelters: A UK Reprise», British Tax Review, núm. 2, pp. 170-208. Institute for Fiscal Studies, Dimensions of Tax Design, Oxford University Press, Reino Unido 2010, disponible en http://www.ifs.org.uk/mirrleesReview. JAMES, S. y NOBES, C. (2007). The Economics of Taxation, Birmingham, Fiscal Publications. KIRCHLER, E. (2007). The Economic Psychology of Tax Behaviour, Reino Unido, Cambridge University Press. McCAFFREY, E. J. (1990). «The Holy Grail of Tax Simplification», Wisconsin Law Review, vol. 5, pp. 1267-1322. McCAFFREY, E. J. (1994). «Cognitive Theory and Tax», UCLA Law Review, vol. 41, pp. 1861-1947. MUMFORD, A. (2002). Taxing Culture: Towards a Theory of Tax Collection Law, Ed. Ashgate, Reino Unido. OECD (1979). «The Taxation of Net Wealth», Capital Transfers and Capital Gains of Individuals. OECD (2008). Study into the Role of Tax Intermediaries. OECD (2010). Tax Policy Reform and Economic Growth. SANDFORD, C. (1995). Taxing Wealth, from More Key Issues in Tax Reform, Inglaterra, Fiscal Publications. THURONYI, V. (2003). Comparative Tax Law, Holanda, Kluwer Law International.

${ }^{6} C f r$ r. TYSON, L. (2013). «La modernización de la tributación de las empresas», 26 de junio, en The Project Syndicate, disponible en http://www.project-syndicate.org/commentary/modernizing-corporate-taxation-by-laura-tyson/spanish. 
Estableciendo que crea valor, por ejemplo I+D, know how, siendo lo que compone la base imponible. Señalando desde el punto de vista cualitativo y cuantitativo, dónde se realizan las operaciones, cómo y cuánto se paga de cuota tributaria, allí donde está su base imponible.

De tal forma que si mueves valor, se mueve la base imponible, no hay un derecho eterno a la base imponible, la base imponible está donde hay creación de valor.

La formación de precios puede ser menos eficiente al haber menos datos, por ello es fundamental la documentación ofreciendo transparencia y seguridad jurídica dentro y fuera del grupo, evitando un mercado ciego. Los precios de transferencia afectan a la reducida empresa, y a las grandes empresas, tal y como reconoce la propia Exposición de Motivos de la Ley 29/2006, de 29 de noviembre, de medidas para la prevención del fraude fiscal, siendo las Directrices en materia de precios de transferencia de la OCDE la guía de referencia para las reglas españolas, como un instrumento esencial para su interpretación ${ }^{7}$.

\subsection{Naciones Unidas}

El Consejo Económico y Social de las Naciones Unidas, por medio de su Resolución número 1.273 (XLIII), de 4 de agosto de 1967, adopta crear el Comité de Expertos, al que le encomienda explorar los medios para facilitar la conclusión de acuerdos fiscales entre países desarrollados y países en proceso de desarrollo sobre bases aceptables para ambos grupos de países, distintas de las que han fundamentado los tratados «concebidos en función de las relaciones entre países desarrollados» buscando «un modelo de acuerdo más apropiado», tal y como se pone de manifiesto en las VI Jornadas Latinoamericanas de Derecho tributario del ILADT en Punta del Este (Uruguay) en 1970. Este Comité de Expertos, en lugar de buscar nuevas bases, decide, pese a la opinión de los representantes latinoamericanos, adoptar como bases de sus tareas el proyecto de Convención Modelo de la OCDE, preparado por expertos de países con un nivel de desarrollo económicamente igual y que descansa sobre el principio de la imposición en el país de la residencia del titular de los beneficios imponibles, lo que no contempla los principios invariablemente sustentados por los países en desarrollo ni coincide con las motivaciones que evidentemente originaron la creación del Comité.

La resolución número 1.430 (LVI), de 6 de junio de 1969, del Consejo Económico y Social autoriza la continuación de los trabajos del Comité de Expertos, recordando «su Resolución 486 B (XVI) del 9 de julio de 1953, en que recomienda que el principio del país fuente constituya el principal fundamento de los acuerdos fiscales».

\footnotetext{
${ }^{7}$ Aunque no toda la doctrina está de acuerdo con este planteamiento, vid. VEGA GARCÍA, A. (2011). «Es obligatorio interpretar la normativa española de origen sobre precios de transferencia según las Directrices de la OCDE?», Crónica Tributaria, núm. 4, pp. 39-56.
} 
El Modelo de Convenio Fiscal de la ONU, así como el Modelo de Convenio Fiscal de la OCDE, han tenido una profunda influencia en la práctica de los tratados internacionales. Ellos forman la base de la mayoría de los tratados entre los países, destinadas a proteger a los contribuyentes contra la doble imposición, con el fin de mejorar el flujo de comercio e inversión internacional, así como la transferencia de tecnología, mientras que conserva la potestad tributaria adecuada a los gobiernos.

El objetivo particular del Modelo de Convenio de 2001 de las Naciones Unidas es facilitar la entrada de tratados fiscales bilaterales de los países en desarrollo, lo que contribuiría a la consecución de sus objetivos de desarrollo. Las similitudes entre los modelos de la ONU y la OCDE reflejan la importancia de lograr la coherencia, mientras que las áreas más importantes de divergencia refleja membresías y prioridades diferentes de las dos organizaciones.

El Modelo de Naciones Unidas en general, mantiene una mayor proporción de ingresos fiscales para el «Estado de origen», el país donde la actividad de inversión o de otro tipo se lleva a cabo. Mientras que el modelo de la OCDE mantiene una mayor participación al «Estado de residencia», el país del inversionista, empresario. Por tanto, el Modelo de Naciones Unidas normalmente permite más derechos de imposición a los países en desarrollo sobre los ingresos generados por las inversiones extranjeras en estos países.

Las principales actualizaciones en 2010 del modelo de la ONU son:

1. que establece el arbitraje obligatorio vinculante, cuando una disputa no puede resolverse en el marco del Procedimiento de Acuerdo Mutuo de costumbre;

2. confirma y aclara la importancia del intercambio de información bajo el Modelo de Naciones Unidas;

3. que establece las normas bajo las cuales los Estados pueden ayudarse mutuamente en la recaudación de impuestos;

4. se dirige a la evasión de impuestos relacionados con la posible imposición de las plusvalías, y

5. se trata de ingresos por servicios personales independientes en línea con el modelo de la OCDE.

\subsection{El rol de la $O C D E$}

La OCDE está ganando la exclusividad en aras de un mayor protagonismo como agente colaborador a nivel fiscal.

$\mathrm{La} \mathrm{OCDE}^{8}$ ha pasado por distintas fases, en cuanto a precios de transferencia se refiere:

${ }^{8}$ El 21 de junio 1976 se adaptaron por primera vez las Líneas Directrices de la OCDE (en adelante, las Directrices), como parte de la Declaración sobre Inversión Internacional y Empresas Multinacionales. El objetivo principal de las Directrices era garantizar que las actividades de las EMN se desarrollaran en armonía con las políticas nacionales de los países de la OCDE y fortalecer la base de la confianza mutua entre las empresas y las autoridades gubernamentales. Las Líneas Directrices de la OCDE para Empresas Multinacionales son 
1. fase, 1995-1998: Desarrollo de las Directrices en materia de precios de transferencia.

2. fase, 1999-2003: Proyectos largos y complejos: subcapitalización, opciones sobre acciones.

3. ' fase, desde 2004: presión por completar las Directrices en materia de precios de transferencia; incremento de las relaciones con países no miembros de la OCDE: Asia, Latinoamérica, Europa del Este. Mayor comunicación y vinculación con la comunidad empresarial. Incremento de países observadores, acercamiento de países no OCDE. Contribuyendo también en los grupos de trabajo de Naciones Unidas. Conscientes de ello, la OCDE colabora con: Brasil $^{9}$, Rusia ${ }^{10}$, Indonesia, China ${ }^{11}$, y Sudáfrica ${ }^{12}$. Tomando protagonismo países como India $^{13}$, México ${ }^{14}$, Perú $^{15}$, Venezuela ${ }^{16}$, Colombia $^{17}$, Argentina $^{18}$.

Realizando los proyectos en los que interviene con un desarrollo práctico, por ejemplo en materia de análisis de comparabilidad, análisis funcional, examen de las transacciones, el entorno del grupo del cual forma parte la entidad. También en cuanto a pérdida de protagonismo de la OCDE, se ha visto empañada por la creación en 2001, bajo la presidencia española de turno de la UE de la creación del Foro Conjunto en materia de PT, con el informe de mayo de 2005, y su propuesta de código

recomendaciones dirigidas por los gobiernos a las empresas multinacionales. Los 30 miembros de la OCDE y 9 países no-miembros -Argentina, Brasil, Chile, Eslovenia, Rumania, Estonia, Israel, Letonia y Lituaniahan suscrito las Directrices. Las Directrices enuncian principios y normas voluntarias para una conducta empresarial responsable en terrenos como la fiscalidad. URL: http://oecdwatch.org/sobre-ocde/guidelines .

9 Vid. MACHADO, C. y GUTIÉRREZ BURGOS, P. (2006). «Brazil», Tax Planning International Special Report, abril 2006, pp. 17-18.

10 Vid. TISSOT, D. (2006). «Russia», Tax Planning International Special Report: Tranfer Pricing Documentation, abril 2006, pp. 56-58.

11 Vid. AA. VV. (2011). Transfer Pricing and Cross-Border Inter-Company Transactions, China, Asia Briefing. AA. VV. (2011). Transfer Pricing in China, China, Asia Briefing; AA.VV. (2013). China Business Handbook, US Commercial Service, China, Asia Briefing, disponible en http://export.gov/china/build/ groups/public/@eg_cn/documents/webcontent/eg_cn_055956.pdf/.Vid. EICHELBERGER, J. y TAO, P. (2006). «China», Tax Planning International Special Report, abril 2006, pp. 25-26.

12 Vid. ERASMUS, D. N. (2009). Transfer Pricing Workshop Incorporating - How to interact with the South African Revenue Service and Ensure that your constitutional rights are upheld, International Tax Institute Veritas, Thomas Jefferson School of Law, Research Paper No. 1481223, 4 de octubre de 2009, disponible en http://ssrn.com/abstract=1481223. Vid. GROVÉ, M. (2006). «South Africa», Tax Planning International Special Report: Tranfer Pricing Documentation, abril 2006, pp. 59-61.

${ }^{13}$ Vid. POPAT, K. (2006). «India», 04/06 Tax Planning International Special Report: Transfer Pricing Documentation, pp. 37-40.

${ }^{14}$ CURIEL GARCÍA, M. (2006). «México», 04/06 Tax Planning International Special Report: Transfer Pricing Documentation, pp. 48-49.

15 Vid. PARGA ECHEVERRI, A. (2006). «Perú», Tax Planning International Special Report: Transfer Pricing Documentation, abril 2006, p. 53.

16 Vid. SÁNCHEZ, G. (2006). «Venezuela», Tax Planning International Special Report: Tranfer Pricing Documentation, abril 2006, pp. 72-71.

17 Vid. FERNÁNDEZ CHIU, A. (2006). «Colombia», Tax Planning International Special Report, abril 2006, p. 27.

$18 \mathrm{Vid}$. BONELL, R. (2006). «La documentación de los precios de transferencia en Argentina», Anuario Jurídico y Económico Escurialense, vol. XXXIX, pp. 259-274. 
de conducta ${ }^{19}$ de 7 de noviembre de $2005^{20}$, la Comunicación tiene por objeto dar cuenta de los trabajos realizados por el Foro conjunto de la UE sobre los precios de transferencia (FCPT) entre enero de 2004 y mayo de 2005 en relación con la documentación sobre los precios de transferencia exigida de las empresas asociadas en la UE, y presentar las conclusiones que ha extraído la Comisión de este trabajo. En la prolongación de su estudio sobre la fiscalidad de las empresas en el mercado interior1, en su Comunicación de 2001 titulada «Hacia un mercado interior sin obstáculos fiscales.

\subsection{El objetivo debe ser la eliminación de la doble imposición}

Una estrategia para dotar a las empresas de una base imponible consolidada del impuesto sobre sociedades para sus actividades a escala comunitaria», la Comisión propuso la creación de un «Foro conjunto de la UE sobre los precios de transferencia». En sus conclusiones de 11 de marzo de 2002, el Consejo se congratulaba de esta iniciativa. Oficialmente creado por la Comisión en junio de 2002, el Foro está compuesto por un experto de la Administración tributaria de cada Estado miembro y diez expertos del sector empresarial. Asisten a las reuniones, en calidad de observadores, representantes de los países candidatos a la adhesión y de la Secretaría de la OCDE. Tal como se indica también en las conclusiones del Consejo, el FCPT, cuyos métodos de trabajo deben basarse en el consenso, tiene por tarea determinar soluciones pragmáticas no legislativas a los problemas prácticos que se plantean en la UE para aplicar las Directrices aplicables en materia precios de transferencia de la OCDE. El trabajo del FCPT refleja claramente la importancia de la aplicación efectiva del Convenio de Arbitraje, en materia de precios de transferencia ${ }^{21}$. Actualmente sigue trabajando hasta el 2019. Los cometidos del grupo serán los siguientes:

1. crear un espacio en el que los expertos de las empresas y de las administraciones fiscales nacionales puedan debatir sobre los problemas relacionados con los precios de transferencia, que obstaculizan las actividades empresariales transfronterizas en la Unión;

2. asesorar a la Comisión en las cuestiones fiscales relacionadas con los precios de transferencia;

\footnotetext{
${ }^{19}$ Soft law a nivel comunitario que sí reúnen las características propias de las backdoor rules. De hecho, la propia Comisión de la UE, a través de su informe de 23 de mayo de 2001, ha reconocido que la utilización de enfoques no legislativos o «legislación blanda» (soft law) podría ser un medio adicional para progresar en el ámbito fiscal; existen ya varios instrumentos comunitarios de soft law como el Código de Conducta sobre Fiscalidad empresarial de 1997 o la Recomendación de la Comisión 94/79, sobre tributación de transfronterizos comunitarios. Vid. COMISION UE (2001). Política Fiscal en la VE. Prioridades para los próximos años, COM (2001) 260 final, pp. 21-22.

20 http://eur-lex.europa.eu/LexUriServ/LexUriServ.do?uri=COM:2005:0543:FIN:ES:PDF.

21 http://ec.europa.eu/taxation_customs/taxation/company_tax/transfer_pricing/arbitration_convention/index_en.htm.
} 
3. ayudar a la Comisión a hallar soluciones prácticas, compatibles con las Directrices aplicables en materia de precios de transferencia a empresas multinacionales y administraciones tributarias de la OCDE, a fin de lograr una aplicación más uniforme de la normativa sobre precios de transferencia en la Unión.

La máxima es eliminar la doble imposición por el principio de libre concurrencia, para ello tendremos en consideración los convenios para evitar la doble imposición, que recogen en términos prácticamente iguales los Modelos de Convenio de la OCDE sobre la renta y el patrimonio, de las Naciones Unidas, y de los Estados Unidos. Dicho principio está desarrollado en el artículo 9 de estos Modelos en relación con la tributación de los beneficios empresariales, se recoge también por otras normas de los Modelos en relación con otros tipos de rendimientos. También queda recogido la utilización de los métodos, métodos clásicos y métodos de último recurso.

Con el objetivo de facilitar la aplicación de las normas la OCDE en 2006 pone en manos de todos el manual para la aplicación de las disposiciones relativas al intercambio de información con fines tributarios ${ }^{22}$, en 2007 el manual para la aplicación efectiva del procedimiento amistoso ${ }^{23}$, junto con el interés creciente en la potenciación e incorporación del Arbitraje.

Estamos ante un incremento de las operaciones económicas internacionales, siendo la resolución alternativa de conflictos la fórmula que cubra el vacío creado por la inexistencia de un derecho tributario global, que sea capaz de dirimir con imparcialidad y eficiencia las divergencias entre los contribuyentes con intereses en distintos países, y la Administración tributaria.

Antes de que se produzcan las discrepancias existe la posibilidad de utilizar como medida preventiva de resolución de conflictos los acuerdos previos de valoración, ofreciendo certidumbre y ofreciendo las previsiones sobre el tratamiento de las operaciones vinculadas, dando seguridad jurídica con el régimen tributario establecido.

22 Vid. DOURADO,A.P. (2013). «Exchange of Information and Validity of Global Standards in Tax Law: Abstractionism and Expressionism or Where the Truth Lies», EUI Working Paper RSCAS 2013/11 European University Institute, Robert Schuman Centre for Advance Studies, Global Governance Programme, Italia 2013, disponible en http://cadmus.eui.eu/handle/1814/26059. El objetivo es el intercambio de información para evitar la erosión de bases imponibles, http://www.oecd.org/tax/exchange-of-tax-information/41814482. $p d f$. En este sentido se enmarca FATCA, Foreign Account Tax Compliance Act, marzo 2010, http://www.irs. gov/Businesses/Corporations/Foreign-Account-Tax-Compliance-Act-(FATCA). Potenciándose los sistemas multilaterales de intercambio de información, en la UE, a través de la Directiva de cooperación administrativa de intercambio de información, Directiva 2011/16/UE del Consejo, de 15 de febrero de 2011, relativa a la cooperación administrativa en el ámbito de la fiscalidad y por la que se deroga la Directiva 77/799/CEE, del Consejo, de 19 de diciembre de 1977, relativa a la asistencia mutua entre las autoridades competentes de los Estados miembros en el ámbito de los impuestos directos y los impuestos sobre las primas de seguros. Dentro de la lucha contra el fraude fiscal. Disponible en http://europa.eu/legislation_summaries/taxation/ fi0006_es.htm, 17 de julio de 2013, 20:46H. PM. Vid. KLEUTGENS, I., KÖHLER, S. (2011) "U.S. -Germany_FATCA - Tax Risks for U.S. Investments: The German Perspective, en WTE Practical European Tax Strategies, EE.UU., Thomson Reuters, octubre 2011, vol. 13, núm. 10, pp. 3-5.

${ }^{23}$ http://www.ief.es/documentos/recursos/publicaciones/libros/OCDE/19_ProcedimientoAmistoso.pdf. 
Siguiendo a Colin Clavey ${ }^{24}$ sobre las previsiones que nos deparará el futuro en la materia: «With the introduction of transfer pricing regimes in countries throughout the world, MNEs are faced with the costs and effort needed to establish arm's length transfer pricing, as well as the burden of documenting and, in some cases, defending their pricing. Tax administrations are also faced with the cost of administering those rules. One area that the OECD may need to consider further is how the application and administration of transfer pricing rules can be carried out as efficiently and effectively as possible, without giving rise to unduly burdensome compliance costs. These are areas that are at least partly considered in Chapter IV of the Transfer Pricing Guidelines. For example, further consideration might be given to how countries select which transfer pricing issues and cases to take up for audit and enquiry. Other issues that might be considered are how administrative arrangements such as advance pricing agreements and safe harbours might make the tasks of complying with and administering transfer pricing rules easier. It is well documented that transfer pricing has become, and continues to be, one of the most important, and perhaps most challenging, tax issues facing both MNEs and tax administrations. Transfer pricing is a dynamic topic and new issues will continue to arise. The OECD will continue its programme of monitoring existing guidance contained in the Transfer Pricing Guidelines to ensure that it continues to meet the needs of tax administrations and taxpayers in a changing world.»

\section{Concepto general de precio de transferencia}

Definición.-José Antonio Gonzalo y Jorge Tua ${ }^{25}$ indican que: «son precios de transferencia los acordados para la cesión de productos o servicios de una empresa a otra filial o dependiente, y también los acordados para la liquidación de operaciones entre sucursales, secciones o departamentos de una misma empresa».

El Tribunal Supremo, en STS de 19 de enero de 1996, los define como «aquellos (precios) que se utilizan o pactan entre si sociedades sometidas al mismo poder de decisión, circunstancia que permite a través de los precios convenidos entre ellas, transferir beneficios o pérdidas de unas a otras, situadas las más de las veces en países distintos». Esta definición cabe también para los precios de transferencia entre sociedades vinculadas en el ámbito nacional.

Atendiendo a estas definiciones hay que documentar la determinación de la valoración con la cuantía resultante del precio de transferencia, teniendo en cuenta los criterios de actuación de las Administraciones tributarias.

${ }^{24}$ Vid. CLAVEY, C. (2008). «Current OECD initiatives», Tax Planning International Transfer Pricing, EE.UU., BNA International, p. 8.

${ }^{25}$ GONZALO, J. A. y TUA, J. (1988). Introducción a la contabilidad internacional, Madrid, Instituto de Planificación Contable, p. 182. 
En operaciones dentro de un mismo grupo los precios o contraprestaciones no necesariamente se establecen con base a precios de mercado, la base imponible de las entidades que forman parte de un grupo de entidades vinculadas está en función de su política de precios de transferencia.

En ausencia de regulación sobre dichos precios, las Administraciones fiscales de un país están en desventaja para asegurar la tributación conforme a una base imponible justa y equilibrada.

Según Müller, Gernon y Meek $^{26}$, la necesidad de determinar un precio de transferencia se presenta cuando se intercambian bienes y servicios entre unidades de la misma compañía, por ejemplo: cargos por servicios administrativos y gerenciales, regalías por derechos intangibles, transferencias de artículos terminados para reventa y cargos por servicios técnicos.

Un precio de transferencia es un sustituto del valor de mercado, se usa cuando una subsidiaria de una corporación le vende a otra. El sistema de precios de transferencia establece un valor monetario sobre los intercambios en las operaciones vinculadas. El precio establecido es registrado por el vendedor como un ingreso y por el comprador como un costo.

Siguiendo a Trapé 27 , los precios de transferencia son la «expresión acuñada en la doctrina y práctica internacional con la cual se quieren designar los precios a los cuales las empresas asociadas o vinculadas han de valorar las transacciones efectuadas entre ellas. Es principio aceptado internacionalmente que dichas transacciones deben valorarse al precio que hubieran aplicado empresas económicamente independientes entre sí en condiciones idénticas o muy similares a las acontecidas en relación con las operaciones que se están valorando».

Por precios de transferencia entendemos los precios que se aplican en transacciones intragrupo, es decir entre entidades asociadas o vinculadas, los cuáles deben adaptarse al mercado. Estas transacciones pueden ser:

- Bienes tangibles: productos terminados, materias primas y componentes, equipos, ingredientes activos, productos terminados.

- Servicios: servicios administrativos, apoyo a las ventas y comercialización, apoyo a la gestión, informáticos, investigación y desarrollo, manufactura por contrato, empaquetado y etiquetado.

- Propiedad intelectual: derechos de marca, uso de marca o nombre, patentes, tecnología, royalties.

- Operaciones financieras: gestión de tesorería, préstamos/aplicaciones intragrupo, cuentas por cobrar dentro de la entidad, netting -compensación neta de créditos y débitos-, clearing -préstamos.

${ }^{26}$ MÜLLER, G. G.; GERNON, H. y MEEK, G. (1997). Accounting: An International Perspective, Chicago, Richard D. Irwin, p.180.

27 TRAPÉ, M. (2007). «Los precios de transferencia», Manual de Fiscalidad Internacional, vol. II, Madrid, IEF, p. 1029. 


\subsection{Origen}

Los precios de transferencia tienen su origen y desarrollo en el siglo $\mathrm{xx}$, durante el cual fluye la expansión de las empresas multinacionales tal y como las conocemos hoy, las cuales fueron implantando estrategias ${ }^{28}$, políticas de precios, para planificar sus beneficios, minimizando los riesgos a través de la diversificación de las actividades productivas, productos complementarios ${ }^{29}$, prácticas comerciales, operaciones financieras, conscientes del valor estratégico incorporándolo a sus bienes y servicios, de esta forma surge como técnica de planificación los precios de transferencia entre las distintas entidades que conforman un mismo grupo multinacional.

Los precios de transferencia aparecen por primera vez durante la Primera Guerra Mundial, utilizando una legislación simple, comenzando por aplicar unos principios preventivos más que operativos, dada la situación de la economía en esa época. Gran Bretaña fue el pionero en aplicar los precios de transferencia en 1915, aunque realmente quién los implementó fue Estados Unidos en el año 1917. Estados Unidos incorpora el primer dispositivo legal referido a los precios de transferencia en 1921, y en el año 1968 elabora las normas que establecen los métodos de determinación de los precios de transferencia ${ }^{30}$, que permiten asignar las ganancias a cada entidad controlada por los mismos intereses. Entre los años 1915 hasta mediados de 1960, se comienza a utilizar en las legislaciones los precios de transferencia, aunque su utilización no ha sido generalizada.

Una vez que la economía mundial se fortalece tras la Segunda Guerra Mundial, empiezan a tratarse los precios de transferencia y se les dota de una gran relevancia, hasta tal punto que en la actualidad, los precios de transferencia se han convertido en una de las cuestiones que más preocupan a las empresas en el ámbito de la fiscalidad directa.

\subsection{La autonomía de las partes}

La autonomía de las partes para establecer una estructura de cadena de valor en cualquier territorio -Supply chain restructuring ${ }^{31}$ - es o debe ser un derecho global,

${ }^{28}$ LLINARES, E. y GONNET, S. (2006). Gestion Stratégique des Prix de Transfert, París. EFE.

29 Vid. CASLEY, A. y WEBB-MARTIN, L. (2003). «Application of Set-Offs in Transfer Pricing Disputes», International Transfer Pricing Journal, mayo-junio 2003, Ámsterdam, IBFD, pp. 104-105. Resaltan los casos de las empresas de telefonía con los terminales y los contratos de permanencia, cobertura, y por otro lado el caso de Gillette, maquinillas de afeitar y las cuchillas.

30 Vid. UCKMAR, V. (1999). Corso di Diritto Tributario Internazionale, Verona, CEDAM, p. 9. En las Directrices aplicables en material de precios de transferencia a empresas multinacionales y administraciones tributarias, en el capítulo I al final, introduce los métodos previstos para valorar si las operaciones vinculadas son conformes con el principio de libre concurrencia. En el capítulo II se estudian los métodos tradicionales, basados en la transacción, que son el método del precio libre comparable, el método del coste incrementado y el método del precio de reventa. Añade el capítulo III dos métodos basados en el beneficio, que son el método de la distribución del resultado y el método del margen neto del conjunto de las operaciones.

31 Vid. HENSHALL, J. (2008). «Supply chain restructuring: IP transfer pricing and taxation», Tax Planning International Transfer Pricing, núm. 5/08, pp. 1-4. 
necesario, fruto de la globalización ${ }^{32}$, siguiendo al profesor Antonio Sánchez-Bayón (Introducción al Derecho comparado y global. Teorías, formas y prácticas, Delta, Madrid, 2011,p. 59): «Derecho positivo vivo, a armonizar, uniformar y unificar, para su mayor y mejor adecuación a la realidad en curso, como es la globalización» ${ }^{33}$. Origina que los contribuyentes, ciudadanos y entidades, tengan el reconocimiento a establecerse y realizar sus operaciones allí dónde le son menos gravosas, dentro de una política liberal internacional generadora de progreso en todos los confines del planeta. Todo en el marco de un derecho global que permita equilibrar y armonizar sistemas jurídicos sin uniformarlos, dotándolos de la seguridad jurídica que requiere todo contribuyente, persona física o jurídica, con asunción de principios que le ofrezcan transparencia y seguridad en la planificación ${ }^{34}$, dentro de los parámetros que marca la OCDE, soft law de validez global. En ocasiones, el término precio de transferencia ${ }^{35}$ es usado con un sentido peyorativo ${ }^{36}$, para denotar el traslado de la

32 Vid.CALDERÓN CARRERO, J. M. (2007). «The OECD transfer pricing guidelines as a source of tax law: is globalization reaching the tax law?», Intertax, vol. 35, núm. 1, pp. 4-29.

33 Bajo la denominación Derecho Comparado y Global (DCG) se alude a la rama jurídica de las encrucijadas y entrelazamientos, aquella que permite reintegrar el derecho, tendiendo puentes entre sus diversos Ordenamientos, cuyas relaciones se han intensificado con la globalización - ya no se está limitado por las fronteras de los Estados-nación, sino por el alcance real de las relaciones jurídicas y sus normas. Se procura así, a través del DCG, tanto el encauzamiento de las nuevas reglas de ordenación social, como la resolución de posibles conflictos inter y transordinamentales, además de propiciarse el avance en los procesos de armonización, uniformización y unificación del emergente derecho común o (neo) utrumque ius de rótulo sincrético ilustrativo de su naturaleza, impulsado en gran medida por las organizaciones internacionales.

34 Vid. AA.VV. (2011). Implementing the Tax Transparency Standards: A Handbook for Assessors and Jurisdictions, París, OCDE.

35 ARESPACOCHAGA, J. (1998). Planificación Fiscal Internacional, Madrid, Marcial Pons, pp. 226 y ss. «[...] La OCDE viene desarrollando decisivas iniciativas en orden a conseguir un consenso entre los Estados miembros en el tratamiento fiscal de los "precios de transferencia". Históricamente tras el Informe de 1979 (Transfer Pricing and Multinational Enterprises), ya consolidado en su aplicación, le siguió el Informe de 1984 (Transfer Pricing and Multinacional Enterprises: Three Related Issues). A partir de 1993 se inician los trabajos tendentes a lograr un concepto de precios de mercado o precios entre empresas independientes, arm's length principle, aceptable tanto para las autoridades fiscales de cada uno de los Estados. El contenido de esta revisión se divide entre tres partes diferenciadas:

- Parte I, que analiza los principios generales relativos a los precios de transferencia y a los métodos aplicables:

-Se reafirma el en el criterio de precios entre empresas independientes, arm's length principle. Admite, en circunstancias excepcionales, el criterio de comparación de beneficios, Comparable profits method, a diferencia de los Estados Unidos (IRS Final Regulations Section 482, 1 julio 1994), que enfatizan el uso de este último como fórmula de tratamiento de los precios de transferencia.

- Se flexibiliza la determinación, en cada caso, del precio de mercado de transacción entre empresas independientes, lo que puede volverse en perjuicio del contribuyente al diluirse la obligación de la carga de la prueba por parte de la Administración.

- Parte II, que analiza la aplicación de los anteriores en las áreas donde aquélla resulte más conflictiva: intangibles y prestación de servicios.

- Parte III, que considera aspectos concretos de aquella aplicación, como la subcapitalización.

[...] Los Estados Unidos se mostraron desde el principio muy críticos con los métodos propuestos por la OCDE, sobre todo cuando no existen transacciones comparables con aquella cuyo precio se pretende ajustar. Como solución alternativa, el IRS introdujo el 1 de marzo de 1991 el método de acuerdos previos en los casos de empresas vinculadas y de precios de transferencia.»

${ }^{36}$ GOTA LOSADA, A. (1987). Relaciones Fiscales Internacionales, Madrid: Instituto de Estudios Fiscales, pp. 50-56. Las operaciones internas entre las distintas unidades que integran la empresa multinacional pueden realizarse al margen de las leyes de mercado porque sus precios se planifican por la autoridad central. 
renta imponible desde una compañía perteneciente a una empresa multinacional, ubicada en una jurisdicción de impuestos elevados, a una compañía perteneciente al mismo grupo, existente en una jurisdicción con bajos impuestos, mediante precios de transferencia incorrectos ${ }^{37}$, a fin de reducir la carga tributaria global del grupo de modo artificioso ${ }^{38}$ y manipulado ${ }^{39}$. Según Victoria Eugenia Combarros Villanueva: «con frecuencia, por ello, se entiende que esta norma trata de evitar la evasión de beneficios, atribuyéndole incluso un carácter penalizador» ${ }^{40}$.

Sin embargo, el ámbito de aplicación de las operaciones vinculadas no debe verse exclusivamente desde el punto de vista de la posible evasión fiscal, sino como medida de fomento de las relaciones internacionales y, por tanto, como mecanismo preventivo que evite riesgos fiscales de doble imposición y, así, de acicate al principio de seguridad jurídica que permita utilizar los precios de transferencia como instrumento de planificación fiscal internacional ${ }^{41}$.

Según Alfonso Gota Losada, los precios de transferencia obedecen a las causas siguientes:

- Evitar, en la medida de lo posible, los efectos del control de cambio de algunos países.

- Facilitar la repatriación de beneficios.

- Paliar el intervencionismo administrativo.

- Eludir o disminuir la carga fiscal.

Compartimos con Alfonso Gota Losada que «la actuación supranacional de las empresas multinacionales, la estrategia ${ }^{42}$ mundial con que operan y el fin de conse-

Estos precios se denominan precios de transferencia (transfer prices) y plantean serios problemas a las Administraciones tributarias de los Estados en que operan, porque a través de ellos, y por multitud de razones, transfieren beneficios de unas sociedades a otras, en perjuicio de los Estados en que residen tales sociedades. Vid. TANZI, V. (2000) “Se necesita una organización mundial de tributación?”, en La Tributación en un contexto de globalización económica, XXXII Asamblea General del C.I.A.T. (Centro Interamericano de Administraciones Tributarias), Madrid: IEF, pp. 72-74.

${ }^{37}$ DAWSON, P. C. y MILLER, S. M. (2209). «International Transfer Pricing for Goods and Intangible Asset Licenses in a Descentralized Multinational Corporation: Review and Extensions», Working Paper Series, Estados Unidos, 22 de enero de 2009. Disponible en internet, 25/07/2011, 13:10 H. PM., en: http:// papers.ssrn.com/sol3/papers.cfm?abstract_id=1331582.

38 UCKMAR, V. (1999). Corso di Diritto Tributario Internazionale, Verona, CEDAM, p. 9.

39 Ibíd., p. 23.

40 COMBARROS VILLANUEVA, V. E. (1988). Régimen tributario de las operaciones entre sociedades vinculadas en el impuesto sobre sociedades, Madrid, Tecnos, p. 19.

${ }^{41}$ BOUZAS, M. A.; COLLADO YURRITA, M. A. y LÓPEZ DE HARO, R. (2007). «Pasado, presente y futuro del régimen de los precios de transferencia en España», AA.VV., Fiscalidad internacional, Madrid, CEF, pp. 639 y 653 y ss. GARCÍA PRATS, F. A. (2005). «Los precios de transferencia: su tratamiento tributario: desde una perspectiva europea», Crónica Tributaria, núm. 117, p. 34. ID. «Cláusulas antielusión en el Impuesto sobre Sociedades: los precios de transferencia». AA. VV. La Lucha contra el fraude fiscal. Estrategias nacionales y comunitarias. Atelier, Barcelona 2008, p. 169.

${ }^{42}$ El estudio de la firma Ernst \& Young (1999), sobre las prácticas, percepciones y tendencias sobre las políticas de precios de transferencia en 19 países, detectó que la mayoría de las empresas multinacionales y sus ejecutivos asocian a este tema, la máxima importancia, para sus estrategias competitivas y decisiones empresariales. 
guir el máximo beneficio posible, hace que entre otros muchos objetivos destaque el lograr la menor presión fiscal» dentro de lo que es la economía de opción, buscando jurisdicciones tributarias con sistemas impositivos coordinados, consistentes, confiables, donde esté garantizada la seguridad jurídica, siendo la actuación de la Administración Tributaria predecible, porque todo esté protocolizado, facilitando la planificación tributaria. Los riesgos son manejables.

\subsection{La elaboración de la documentación ${ }^{43}$}

Para Verónica Grondona ${ }^{44}$, los precios de transferencia deberían ser vistos más bien como un proceso, siendo el input de este proceso la manera en que una compañía es manejada, y siendo el output un sistema de precios de transferencia manejable y defendible.

Los diferentes pasos de este proceso serían: $a$ ) Identificar dónde genera rendimientos la empresa: ¿lo hace a través de sus actividades de producción? o ¿es la marca la que genera las ganancias en la organización? b) ¿Cómo puede una multinacional diseñar un sistema de precios de transferencia apropiado? ¿Cómo puede una multinacional distribuir sus ganancias entre los diferentes actores de su cadena de valor? Esto depende de los roles y responsabilidades de cada parte. No sería justo que si el distribuidor es quien determina los volúmenes de sus mercados se trasladara esta responsabilidad a la planta. Estos son el tipo de roles y responsabilidades que una multinacional necesita tener en cuenta a la hora de decidir cuál de los métodos recomendados por la Organización para la Cooperación y Desarrollo Económico (precio comparable, coste incremental, precio de reventa, distribución de beneficios), o diseñado por ella misma, debe utilizar para compensar a cada una de las entidades de la multinacional. El paso $c$ ) consiste únicamente en poner todo lo antedicho sobre papel, y eso es lo que comúnmente se conoce con el nombre de documentación. Es casi un producto residual de los pasos $a$ ) y $b$ ) descritos.

Siguiendo a Verónica Grondona, si una multinacional ha definido ya un sistema de precios de transferencia para ser utilizado dentro de su organización, puede utilizar luego el Masterfile para documentar este sistema. ¿Cómo tendría que hacerse?: identificando los procesos de negocios dentro de la multinacional, diseñando el sistema de precios de transferencia que será utilizado para determinar las ganancias de cada una de las entidades dentro de la cadena de valor o proceso de negocio y preparando el Masterfile para documentar cada proceso de precios de transferencia.

${ }^{43}$ BONELL COLMENERO, R. (2016). Operaciones vinculadas los deberes de documentación, Valladolid: Lex Nova.

${ }^{44}$ GRONDONA, V. (2011). «El uso del sentido común», Cinco Días, Madrid, 29 de diciembre de 2006. Disponible en internet, 25 de julio de 2011, 13:20H PM., en http://www.cincodias.com/articulo/opinion/uso/ sentido/comun/cdscdi/20061229cdscdiopi_6/Tes/\#. 
El sistema seguido habitualmente por las Administraciones tributarias para no verse lesionadas por estas prácticas ${ }^{45}$ es la de considerar que dichas operaciones deben de valorarse, a efectos fiscales, por el precio que se hubiera pactado entre partes independientes, en un mercado donde existiese libre competencia.

\section{Configuración normativa}

El Derecho Tributario es una disciplina que tiene por objeto de estudio el ordenamiento jurídico que regula el establecimiento y aplicación de los tributos. Siendo una rama del Derecho público, dentro del Derecho Financiero, que estudia las normas jurídicas a través de las cuales la Administración Tributaria ejerce su potestad con el propósito de obtener de los contribuyentes, personas físicas y jurídicas, recursos que sirvan para sufragar el gasto público para la consecución del interés común. Por su incidencia dentro de la actividad financiera, por su coactividad y por la homogeneidad de su regulación, ha adquirido un tratamiento sustantivo.

El contenido del Derecho Tributario se divide en una parte general, que comprende los aspectos materiales, que estudia qué es el tributo, cómo y por qué se establece y regula y quién lo establece, y los de naturaleza formal o procedimental analiza la aplicación efectiva del tributo y, por tanto, los procedimientos de aplicación de los tributos. En conexión con los principios tributarios constitucionales, las fuentes de las normas, la aplicación temporal y espacial, la interpretación de las normas, la clasificación de los tributos y sus características, los métodos de determinación de las bases tributarias, las infracciones y sanciones tributarias, la extinción de la deuda, los procedimientos de recaudación, inspección y revisión de los actos administrativos y el estudio de los órganos de la Administración Tributaria, mientras que el contenido de la parte especial se centra en las disposiciones específicas de cada uno de los tributos que componen el sistema fiscal, analizando el Derecho Positivo. Se examinan cuestiones como la naturaleza de cada tributo, su hecho imponible, supuestos de no

${ }^{45}$ GARCÍA PRATS, F.A. (2005). «Los precios de transferencia: su tratamiento tributario desde una perspectiva europea», Crónica Tributaria, núm. 117/2005, Instituto de Estudios Fiscales, Madrid 2005, p. 34, señala: «La reacción contra la utilización de los precios de transferencia como mecanismo de deslocalización/ reasignación de rentas entre jurisdicciones fiscales, o como mecanismo de elusión fiscal constituye una de las formas más depuradas con las que cuentan los Estados para asegurar sus exigencias internas de justicia tributaria exigiendo el gravamen por la porción de renta transnacional que les corresponde gravar de forma legítima. Sin embargo, ni constituye el único mecanismo para hacer frente a los problemas de evasión fiscal, ni constituye un mecanismo homogéneo en un análisis tributario comparativo. Por un lado, la regulación tributaria de los precios de transferencia no constituye el más importante y más depurado mecanismo de que disponen las Administraciones tributarias para combatir la evasión fiscal. Junto a la regulación de los precios de transferencia existen otras técnicas utilizadas simultánea o paralelamente y que en la actualidad ostentan igual o mayor protagonismo. Existen algunas técnicas, como la normativa contra la subcapitalización, cuya delimitación con los precios de transferencia aparece en ocasiones confusa. Otros mecanismos para atajar la evasión fiscal internacional y conseguir la equidad tributaria son la transparencia fiscal internacional, o los impuestos de salida - exit taxes-. Existe, finalmente, una importante variedad de mecanismos genéricos de lucha contra el fraude o el abuso fiscal, mediante las que pretende atajarse también la evasión fiscal internacional». 
sujeción y exención, sujetos pasivos, base imponible, base liquidable, tipo de gravamen, deducciones, bonificaciones y gestión del mismo.

El Derecho ha de tener la suficiente flexibilidad para adaptarse al cambio social, y la suficiente estabilidad para aportar seguridad jurídica.

Los requerimientos de documentación en materia de operaciones vinculadas, se sitúan en el marco del derecho material, que se compone de una serie de normas y contra normas que recíprocamente constituyen la base de la documentación de las operaciones vinculadas en materia de valoración con el cumplimiento del valor normal de mercado que deben cumplir los contribuyentes que realicen operaciones vinculadas tal y como establece el art. 18 LIS.

El contribuyente tiene que probar con la documentación todos aquellos hechos que sin negar la relación jurídica como existente y válida, excluyen la pretensión del sujeto pasivo en base a los hechos exteriores de la propia relación. El sujeto pasivo tiene la carga de probar con la documentación, todos los elementos que componen el régimen de las operaciones vinculadas, siendo todos los elementos del supuesto de hecho del art. 18 LIS necesarios para la producción de las consecuencias jurídicas y fiscales, favoreciendo esta al actor por la seguridad jurídica que le aporta.

La documentación se realiza en sintonía con la realidad y el plan de negocio de la empresa, la realidad tributaria se corresponde con la realidad empresarial, dentro de la buena gobernanza, transparencia fiscal, comunicación de la planificación fiscal de la empresa, de tal forma que el cumplimiento de las obligaciones de documentación partimos del propio plan de negocio, del mapa de entidades vinculadas, transacciones, ver qué documentación hay que presentar y cómo presentarla. Se trata de ponerlo en orden, coordinándolo con todos los departamentos, dentro del asesoramiento tributario de la entidad. Siendo la planificación fiscal la que sigue al plan de negocio, ofreciendo el mayor beneficio a la obtención de los recursos propios y ajenos, obteniendo el valor añadido.

El juego del mercado tampoco es justo, ni es obligación del mercado ser justo, el mercado tiene que cumplir las reglas de la competencia, esencial en la economía de mercado cumplir con las reglas que lo regulan, no es obligación del mercado hacer justicia, sino que es obligación del Estado, una necesidad de un sujeto político que redistribuya los bienes básicos del Estado del Bienestar.

La cadena de derechos que ha dado lugar a la Declaración de Derechos Humanos tiene tres etapas, primero vienen los derechos civiles, luego los derechos políticos, y tras estos los derechos sociales. Los derechos civiles lo que reclaman del Estado es que no interfiera tanto en la vida de las personas, que deje a las personas libres, las personas reclaman más libertad para hacer con su vida lo que quieran.

La libertad, como concepto clave, que va unida al poder de decisión del individuo, con su posibilidad de razonamiento superior y su cualidad básica que le define como persona, choca, en el momento de su ejercicio, con la característica social del hombre: la convivencia, dentro de un Estado del Bienestar que todos deseamos como ciudadanos. 


\subsection{Los asesores financieros y tributarios en la planificación empresarial}

Los derechos políticos, al consistir en participación política, ya reclaman una cierta intervención del Estado, que es quien tiene que organizar la participación política, contribuyendo a que sea posible, pero los derechos sociales reclaman la participación aún mayor del Estado; si hay derechos fundamentales, el Estado debe garantizarlos, interviniendo de forma activa, limitando la libertad al intervenir, estableciendo la forma en que hay que redistribuir la renta y la riqueza, income and wealth. El sujeto de la justicia son las instituciones públicas, el poder legislativo, ejecutivo y judicial.

Los asesores deben contar con una información amplia, general y completa de la legislación, usos y costumbres de aquellas operaciones vinculadas, que aporta la documentación de las operaciones, en los países en que tales inversiones tienen lugar o, en su caso, de los países de donde las inversiones provienen. Esta información que ofrece la documentación, en ocasiones, se encuentra al alcance del asesor fiscal de la entidad, pero en otras ocasiones han de recurrir a asesores extranjeros en cualquiera de las etapas de organización del proceso, que ofrece la planificación de las operaciones vinculadas ${ }^{46}$.

Los datos básicos que conforman toda la información, entendida de forma lógica y organizada ${ }^{47}$, que se requiere para elaborar una documentación óptima.

\subsubsection{El conocimiento de la realidad de los hechos}

En los supuestos del asesoramiento tributario internacional, es necesario tener en cuenta muchos más hechos en comparación con la documentación de la planificación de las operaciones vinculadas a nivel nacional. El proceso se vuelve más complejo y los asesores deben tener especial cuidado en asegurarse de que los hechos proporcionados por la entidad son exactos y completos. Hay que tener en cuenta la articulación contable y fiscal.

El tratamiento impositivo, los costes indirectos que conlleva el cumplimiento de la obligación de documentar estas operaciones. En cada operación debe de realizarse un análisis exhaustivo de la documentación a elaborar.

El precio es uno de los principales criterios de compra, existe una gran indiferenciación en la compra venta de bienes y en la prestación de servicios ${ }^{48}$. Nos

\footnotetext{
46 Vid.ALBERT, J. (2008). Study on Effects of Tax Systems on the Retention of Earnings and the Increase of Own Equity, 15 de febrero de 2008. Disponible en internet, 28 de marzo de 2013, 10:33H. AM: http:// ec.europa.eu/enterprise/newsroom/cfl_getdocument.cfm?doc_id=3920.

47 Vid. FRICKÉ, M. (2012). Logic and the Organization of Information, Nueva York, Springer.

48 Vid. KEEGAN, D. P. y HOWARD, P. D. (1988). «Making Transfer Pricing Work for Services», Journal of Accountancy, marzo 1988, EE. UU., pp. 96-103. RIBES RIBES, A. (2012). «Interpretación del concepto "prestaciones de servicios realizadas a título oneroso" (art. 2.1, Sexta Directiva IVA) STJCE de 29 de julio de 2010», Unión Europea, CISS, diciembre, pp. 30-42. Disponible en internet, 30/03/2013, 13:00H. PM: http://www.ciss.es/publico/deloitte/2012_70_S_030.pdf.
} 
encontramos con que la fijación del precio es en sí un factor estratégico para la venta, pero también para el posicionamiento estratégico. ¿Qué se hace en el mercado? ¿Cómo se fijan los precios? ¿Cómo se relaciona con lo que queremos ser? Las reglas que afectan a los precios de transferencia giran en torno al principio Arm's length o principio de plena competencia, fruto de los acuerdos de la OCDE en las Directrices aplicables en materia de precios de transferencia a empresas multinacionales y administraciones tributarias. De tal forma, que bajo el principio Arm's length, las entidades vinculadas se deben comportar con respecto a las transacciones, como si la otra entidad fuera no vinculada, en caso contrario, las Administraciones tributarias tienen el derecho de ajustar los beneficios derivados de las transacciones de las entidades en cuestión, hasta el nivel que hubiera sido justo aplicando el principio Arm's length.

En el ámbito empresarial, bajo el control que realizan la Comisión Nacional de la Competencia ${ }^{49}$, es frecuente que se abran expedientes por pacto de precios, por ejemplo: «La Comisión Nacional de la Competencia abre tres expedientes en el sector cosmético por pacto de precios $»^{50}$; «Multa a Correos por ofrecer servicios bajo el precio de coste ${ }^{51}$; «Multa de 315 millones a Ariel y Skip por pactar precios. Henkel se libra de la sanción por delatar ante Bruselas el cartel del detergente» ${ }^{52}$.

\subsubsection{El deber de contrastar la documentación de las operaciones vinculadas}

No solo la política de precios de las empresas, sino que también los precios de transferencia ocupan un lugar muy destacado en la agenda de los consejeros de administración de entidades ${ }^{53}$, en particular de empresas cotizadas a raíz del art. 529 ter

49 http://www.cncompetencia.es/.

50 Periódico La Razón, sábado 21 de junio de 2008, en la sección de Economía, p. 49, indicaba: «La Comisión Nacional de la Competencia ha iniciado la investigación de tres posibles acuerdos de precios en el sector de los cosméticos, para lo que ha incoado tres expedientes sancionadores y ha realizado trece inspecciones en las principales empresas del sector. Los expedientes sancionadores se refieren a geles de baño y ducha (incoado a Puig Beuty \& Fashion Group, Sara Lee Household \& Body Care España, Henkel Ibérica, Colomer Group Spain y Colgate Palmolive España) y peluquería (L'Oreal España, Wella, Colomer Group Spain, Eugne Perma, Montibello, Lendan, Henkel Ibérica, DSP Haircare Products y Stanpa)».

${ }^{51}$ Periódico $A B C$, miércoles 31 de agosto de 2011, en la sección de Bolsa, p.41, indicaba: «La Comisión Nacional de Competencia ha multado con 4,8 millones de euros a Correos y Telégrafos por prestar servicios postales a grandes clientes a precios que no cubrían los costes. Correos ha anunciado que recurrirá».

52 Periódico El País, Economía, Negocios, Madrid, 13 de abril de 2011: «La multinacionales Unilever, fabricante de Skip, y Procter \& Gamble, productora de Ariel, pagarán una multa de 315 millones de euros que les ha impuesto Bruselas por pactar precios del detergente en ocho países durante tres años. El fabricante Ariel ha recibido la mayor sanción, de 211 millones, y Unilever de 104, mientras que Henkel, que produce la marca Persil y también formó parte de este cartel del detergente, se ha librado de la multa por haber confesado la existencia de esta práctica ilícita a las autoridades de Competencia de la Comisión Europea». Disponible en internet, 17 de septiembre de 2011, 12:26H PM: http://www.elpais.com/articulo/economia/Multa/315/millones/Ariel/Skip/pactar/precios/elpepueco/20110413elpepueco_7/Tes.

${ }^{53}$ Mercedes SERRALDE, "El consejo de las empresas cotizadas será responsable de la política fiscal", en Expansión, 26 de diciembre de 2014, p. 22: "A partir del 1 de enero, el consejo de administración de las cotizadas será responsable de las estrategias tributarias y deberá determinar la política de gestión de riesgos fiscales. Cuando Hacienda imponga una multa millonaria a una empresa o los tribunales establezcan responsabilidades penales por haber diseñado un entramado fiscal ilegal, el consejo de administración no podrá señalar al responsable de Fiscal. A partir del próximo 1 de enero, el propio consejo de administración de 
de la Ley de Sociedades de Capital, RD Legislativo 1/2010, de 2 de julio, que incluye como facultades indelegables del Consejo de Administración: «La determinación de la política de control y gestión de riesgos, incluidos los fiscales, y la supervisión de los sistemas internos de información y control».

La Agencia Tributaria se ha inspirado en la legislación del Reino Unido, desde la última década, se están adoptando normas cuyo objeto es que las grandes empresas lleven a cabo una adecuada gestión de sus riesgos fiscales. Además de la aplicación de las Directrices de la OCDE, marcan las líneas de actuación para establecer la responsabilidad fiscal del consejo de administración.

En EEUU, el antecedente es la Ley Sarbanes Oxley, de 30 de julio de 2002, cuyo artículo 404 impone el deber de adjuntar a los estados financieros el informe de la gerencia sobre la fiabilidad de los controles internos de la compañía. Dicho informe no se limita a la estrategia u operaciones de la empresa, sino que todas las áreas están afectadas, incluyendo por tanto el departamento fiscal.

En consecuencia, se impone una obligación que despliega sus efectos sobre los aspectos tributarios, imponiendo el deber de pronunciarse acerca de la fiabilidad de

las sociedades cotizadas será responsable de todas las estrategias tributarias de la empresa y, por lo tanto, se encargará de determinar la política de gestión de riesgos fiscales. Así lo establece la reforma de la Ley de Sociedades de Capital que entró en vigor el pasado día 24, que contiene una disposición para que este importante aspecto fiscal esté vigente desde enero, de acuerdo con el comienzo del ejercicio de las compañías. De esta forma, Hacienda intenta poner coto a la planificación agresiva y a la ingeniería tributaria de las compañías trasladando la responsabilidad directamente al consejo, que en ocasiones asegura no conocer las decisiones de los responsables de Fiscal. En esta norma, Hacienda ya ha introducido modificaciones sobre el control de la morosidad, con lo que las empresas deberán publicar en sus cuentas anuales e informar a sus accionistas de su plazo medio de pago a proveedores. Gestión de riesgos en lo que respecta a las responsabilidades e información sobre la política tributaria, el consejo de administración deberá diseñar la gestión de riesgos, pues de ello no solo dependen las cuentas financieras de la sociedad, sino también su imagen reputacional que, debe atender no solo a la normativa fiscal nacional sino a la de varias jurisdicciones, trasladan desde Hacienda. Además y como función indelegable, el consejo deberá aprobar las operaciones de especial trascendencia tributaria para reforzar la responsabilidad fiscal. Asimismo, estará obligado a determinar la estrategia tributaria y responsabilizarse de los resultados de las mismas ante los accionistas, asegurando de este modo unas prácticas de gobierno corporativo adecuadas. A esto se suma que el consejo deberá aprobar la creación o adquisición de participaciones en entidades domiciliadas en paraísos fiscales, así como cualesquiera otras operaciones de naturaleza análoga que «pudieran menoscabar la transparencia», dice la norma. Todas estas tareas se convierten en «indelegables» para el consejo de las cotizadas. Estas sociedades deben hacer público con carácter anual un informe de gobierno corporativo. Actualmente el contenido mínimo debe incluir, entre otras cuestiones, los sistemas de control del riesgo de la sociedad, entre los que ahora será obligatorio incluir los riesgos fiscales. También se establece como nueva función de la comisión de auditoría la de supervisar la eficacia de los sistemas de control de riesgo fiscal y la determinación de la estrategia tributaria. Desde Hacienda trasladan que la intención es que las obligaciones se vayan extendiendo a todas las empresas. El Fisco subraya que dará relevancia a la gestión de riesgos fiscales y enmarca los cambios en las mejoras del gobierno corporativo. También hay implicaciones de riesgo reputacional y relación con los esfuerzos para prevenir el blanqueo de capitales. Se hará un esfuerzo de divulgación si es necesario, concede Hacienda.

El escenario actual es que solo el 17 por 100 de las compañías realiza alguna mención a su estrategia fiscal; en el caso del Ibex, el 37 por 100, según PWC. El 18 por 100 considera que el consejo debe involucrarse más en este asunto.

Este cambio sigue el ejemplo de otros países, cuando crece la preocupación ante el traslado artificial de beneficios de las multinacionales para no tributar." Disponible en internet, 26/12/2014, 12:05 PM: http:// www.expansion.com/2014/12/26/economia/1419612250.html? $a=$ VO246blc8a9d0531f55bb2f50be338c$c 33 e \& t=1419765691 \&$ cid=SMBOSO22801\&s_kw=twitter. 
los controles, también los fiscales. Siguiendo esta tendencia, existen disposiciones similares en el Reino Unido, con carácter imperativo, y en Holanda, y Australia, bajo el principio de autorregulación-comply or explain.

En el Reino Unido, los directores financieros deben certificar anualmente que sus sistemas de aplicación de impuestos y control de riesgos fiscales son adecuados, son los correctos.

En Holanda se ha establecido un sistema optativo de relación cooperativa entre la Administración tributaria y las empresas, a través del cual estas asumen el compromiso de comunicar a la Agencia sus riesgos fiscales, mientras que la Administración se compromete a confiar en el buen hacer de los contribuyentes, confianza basada en menores controles y menos exhaustivos, bajo el principio Just Culture, de confianza mutua.

Australia ha establecido un marco detallado de gestión de riesgos fiscales que las empresas que se consideren socialmente responsables deben adoptar ${ }^{54}$.

En Canadá, la Agencia tributaria ha elaborado un sistema para evaluar el perfil de riesgo de las empresas transnacionales, de tal forma que siguiendo el principio Just Culture, las que ofrezcan confianza, transparencia en la trazabilidad de las operaciones y cooperen con la Administración, se les asignará una calificación de riesgo fiscal inferior, en un entorno de control bajo, debido a su cumplimiento.

Todo ello es consecuencia de la práctica internacional, producto de la decisión de los factores que se tienen en cuenta en la producción, renta per cápita, producto interior bruto, paridad del poder adquisitivo, que permite comparar los distintos niveles de vida de diferentes países, y la incidencia con la oferta y demanda en el comercio exterior, puesto que nuestro sistema utiliza los precios para asignar recursos escasos.

\subsection{El asesor y la planificación fiscal}

En la toma de decisiones, ya se sitúa el régimen fiscal de la empresa en la agenda del Consejo de Administración, por lo tanto es preciso que el asesoramiento tributario sea conciso ${ }^{55}$, con la previsión y la provisión de la cuantificación, alcance de responsabilidades y los controles al respecto.

La planificación fiscal se puede definir, de forma concisa, como la forma de optimizar el resultado fiscal a través de fórmulas adecuadas y legales, mediante un proceso en el que se han de tomar en consideración tanto factores fiscales como datos relevantes no fiscales. Asimismo definir el tipo de relación con las Administraciones tributarias.

54 Vid. Goods and Services Tax Governance and risk management guide for large businesses: https://www. ato.gov.au/Business/GST/In-detail/Non-profit-and-government-organisations/Government-organisations/ GST-governance-and-risk-management--Government/. https://www.ato.gov.au/uploadedFiles/Content/ITX/ downloads/how_gst_works.pdf.

55 Vid. ORDOWER, H. (2010). «The Culture of Tax Avoidance», Saint Louis University Law Journal, vol. 55, abril 2010, pp. 47-128. 
En una materia tan técnica y específica que requiere de la publicación de los criterios de aplicación, especialmente en lo referente a valoraciones y el uso de bases de datos comparables, a través de empresas que se dedican a la elaboración de datos para su consulta y redistribución electrónica, que implica la doble transformación de los datos, por un lado documental, y por otro, informática. Los retos y desafíos ante la gestión documental electrónica, la incidencia de las TIC en la efectividad empresarial, el archivo electrónico, la digitalización de las entidades, conservación de la documentación, gestión de documentos, valor probatorio. Eficacia empresarial y eficiencia en el ahorro de costos, traduciéndose en beneficios empresariales para todos $^{56}$.

Si añadimos al concepto anterior el adjetivo internacional, nos encontramos que la misma se da cuando en este proceso aparece algún elemento que tenga algún tipo de implicación fuera de las fronteras nacionales.

\subsection{La relación entre planificación y transparencia}

Está comprometida la entidad con la planificación y la transparencia. Cabe preguntarse también ¿qué tipo de relación existe entre planificación y transparencia fiscal internacional? La transparencia fiscal internacional se presenta como una de las medidas legales que utilizan los Estados con el fin de imputar en el país de residencia rentas acumuladas y retenidas en el extranjero. Es un control que determina la salida de capitales de las entidades e inversores, hacia jurisdicciones que ofrezcan mayores ventajas fiscales.

Nos hallamos, de este modo, en el campo de la fiscalidad internacional contemplada desde dos perspectivas:

\footnotetext{
${ }^{56} \mathrm{Vid}$. Implementación del programa de gestión documental en la empresa, normas de la Organización Internacional de Normalización, ISO 15948, 27000, 30300, 30301, a través de un sistema de gestión, una herramienta que permite optimizar recursos, reducir costes y mejorar la productividad en la empresa. Este instrumento de gestión reporta datos en tiempo real que permitirán tomar decisiones para corregir fallos y prevenir la aparición de gastos innecesarios. Los sistemas de gestión están basados en normas internacionales que permiten controlar distintas facetas en una empresa, como la calidad de su producto o servicio, los impactos ambientales que pueda ocasionar, la seguridad y salud de los trabajadores, la responsabilidad social o la innovación. La ISO 27000 es la norma que explica cómo implantar un Sistema de Gestión de Seguridad de la Información en una empresa. La implantación de una ISO 27000 en una organización permite proteger la información de esta de la forma más fiable posible. Se persiguen 3 objetivos: Preservar la confidencialidad de los datos de la empresa. Conservar la integridad de estos datos. Hacer que la información protegida se encuentre disponible. Una empresa que tiene implantada la ISO 27000 garantiza, tanto de manera interna como al resto de las empresas vinculadas o no, que los riesgos de la seguridad de la información son controlados por la organización de una forma eficiente. El estándar ISO 27000 es totalmente compatible con otras normas de sistemas de gestión (ISO 9001, ISO 14001, OHSAS 18001) y puede ser implantado de forma integrada con estas. Disponible en la red: 7/10/2013, 17:34HPM URL: http://www.aenor.es/aenor/descargadocumento.asp?nomfich=/Documentos/Comercial/Archivos/NOV_DOC_Tabla_AEN_21023_2.pdf\&cd_no$v e d a d=21023 \& c d \_n o v e d a d \_d o c=2$.
} 
- Por un lado, aquella que se refiere a las inversiones españolas en el exterior. Estas están sujetas a contribuir por obligación personal y a las que son de aplicación las medidas para evitar la doble imposición internacional, entre las que se encuentra la medida, considerada unilateral, de la transparencia fiscal internacional.

- Por otro lado, la fiscalidad de las inversiones extranjeras en España. Sometidas a la obligación real de contribuir y sobre las cuales recaen únicamente medidas de tipo bilateral o multilateral.

\section{Referencias bibliográficas}

[1] AHMADOV, J. (2011). «The "Most Appropriate Method" as the New OECD Transfer Pricing Standard: Has the Hierarchy or Methods Been Completely Eliminated?». International Transfer Pricing Journal, vol. 18, núm. 3, IBFD, Amsterdam.

[2] ALBERT, J. (2008). Study on Effects of Tax Systems on the Retention of Earnings and the Increase of Own Equity, 15 de febrero de 2008.

[3] ARESPACOCHAGA, J. (1998). Planificación Fiscal Internacional, Madrid, Marcial Pons.

[4] AVI-YONAH, R. (1995). «The Rise and Fall of Arm's Length: A Study in the Evolution of US International Taxation». Virginia Tax Review, vol. 15, pp. 89-159, EE. UU.

[5] AVI-YONAH, R. (2010). «Between Formulary Apportionment and the OECD Guidelines: A Proposal for Reconciliation». World Tax Journal, vol. 2, núm. 1, IBFD, Amsterdam.

[6] BONELL, R. (2006). «La documentación de los precios de transferencia en Argentina». Anuario Jurídico y Económico Escurialense, vol. XXXIX, pp. 259-274.

[7] BONELL COLMENERO, R. (2016). Operaciones vinculadas los deberes de documentación, Valladolid, Lex Nova.

[8] BOUZAS, M. A.; COLLADO YURRITA, M. A. y LÓPEZ DE HARO, R. (2007). «Pasado, presente y futuro del régimen de los precios de transferencia en España», AA.VV., Fiscalidad internacional, pp. 639-659.

[9] COMBARROS VILLANUEVA, V. E. (1988). Régimen tributario de las operaciones entre sociedades vinculadas en el impuesto sobre sociedades, Madrid, Tecnos.

[10] COOPER, J. y AGARWAL, R. (2011). «The Transactional Profit Methods in Practice: A Survey of APA Reports». International Transfer Pricing Journal, vol. 18, núm. 1, Amsterdam, IBFD.

[11] FRICKÉ, M. (2012). Logic and the Organization of Information, Nueva York, Springer.

[12] KEEGAN, D. P. y HOWARD, P. D. (1988). «Making Transfer Pricing Work for Services». Journal of Accountancy, marzo 1988, pp. 96-103.

[13] GARCÍA PRATS, F. A. (2005). «Los precios de transferencia: su tratamiento tributario desde una perspectiva europea». Crónica Tributaria, núm. 117/2005, Instituto de Estudios Fiscales, Madrid.

[14] GOTA LOSADA, A. (1987). Relaciones Fiscales Internacionales, Instituto de Estudios Fiscales, Madrid, pp. 50-56.

[15] LLINARES, E. y GONNET, S. (2006). Gestion stratégique des prix de transfert, EFE, analyses experts, París. 
[16] ORDOWER, H. (2010). «The Culture of Tax Avoidance», Saint Louis University Law Journal, vol. 55, abril, EE. UU., pp. 47-128.

[17] RIBES RIBES, A. (2012). «Interpretación del concepto “prestaciones de servicios realizadas a título oneroso" (art. 2.1, Sexta Directiva IVA) STJCE de 29 de julio de 2010». Unión Europea, diciembre, pp. 30-42.

[18] TRAPÉ, M., (2007). «Los precios de transferencia». Manual de Fiscalidad Internacional, vol. II, Madrid, IEF.

[19] UCKMAR, V. (1999). Corso di Diritto Tributario Internazionale. Verona, CEDAM. 